This is the peer- reviewed version of the article

Burmudžija AZ, Muškinja JM, Kosanić MM, Ranković BR, Novaković SB, Đorđević SB, et al. "Cytotoxic and Antimicrobial Activity of Dehydrozingerone based Cyclopropyl Derivatives". Chem Biodiversity 2017;14(8) DOI: 10.1002/cbdv.201700077

This article is granted by Ministry of Education, Science and Technological

Development of the Republic of Serbia (Grant Number 172034).

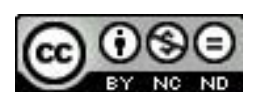

This work is licensed under

Creative Commons - Attribution-Noncommercial-NoDerivative Works 3.0 Serbia 


\section{Cytotoxic and Antimicrobial Activity of Dehydrozingerone based}

\section{Cyclopropyl Derivatives}

Burmudžija Adrijana $Z^{\mathrm{a}}$., Muškinja Jovana $\mathrm{a}^{1}$.,. Kosanić Marijana $\mathrm{M}^{\mathrm{b}}$, Ranković Branislav R ${ }^{\mathrm{b}}$, Novaković Slađana B ${ }^{c}$. Đorđević Snežana B ${ }^{\mathrm{d}}$, Stanojković Tatjana $\mathrm{P}^{\mathrm{e}}$, Baskić Dejan $\mathrm{D}^{\mathrm{f}, \mathrm{g}}$, Ratković Zoran $\mathrm{R}^{\mathrm{a}}$

a Department of Chemistry, Faculty of Science, University of Kragujevac, Radoja Domanovica 12, RS-

34000 Kragujevac,e-mail: wor@kg.ac.rs

$b$ Department of Biology and Ecology, Faculty of Science, University of Kragujevac, Radoja Domanovica 12, RS-34000 Kragujevac Vinca Institute of Nuclear Sciences, University of Belgrade, P.O. Box 522, $\quad$ RS-11001 Belgrade

c Vinča Institute of Nuclear Sciences, University of Belgrade, P.O. Box 522, RS-11001 Belgrade

d National Poison Control Centre, Military Medical Academy, Crnotravska 17, RS-11000 Belgrade

e Institute of Oncology and Radiology of Serbia, Pasterova 14, RS-11000 Belgrade

$f \quad$ Center for Molecular Medicine and Stem Cell Research, Faculty of Medical Sciences, University of Kragujevac, Svetozara Markovica 67, RS-34000 Kragujevac

$g$ Public Health Institute Kragujevac, Nikole Pašića1, RS-34000 Kragujevac

Corresponding author: Bumrudžija, Adrijana Department of Chemistry, Faculty of Science, University of Kragujevac,

Radoja Domanovica 12, RS-34000, Kragujevac

e-mail: wor@kg.ac.rs 


\section{Abstract}

A small series of 1-acetyl-2-(4-alkoxy-3-methoxyphenyl)cyclopropanes was prepared, starting from dehydrozingerone (4-(4-hydroxy-3-methoxyphenyl)-3-buten-2-one) and its $O$-alkyl derivatives. Their microbiological activities toward some strains of bacteria and fungi were tested, as well as their in vitro cytotoxic activity against some cancer cell lines (HeLa, LS174 and A549). All synthesized compounds showed significant antimicrobial activity and expressed cytotoxic activity against tested carcinoma cell lines, but they showed no significant influence on normal cell line (MRC5). Butyl derivative is the most active on HeLa cells $\left(I C_{50}=8.63 \mu \mathrm{m}\right)$, while benzyl one is active against LS174 and A549 cell lines $\left(I C_{50}=\right.$ 10.17 and $12.15 \mu \mathrm{m}$, respectively).

Keywords: Cytotoxic activities, Antimicrobal activities, Dehydrozingerone, Cyclopropyl moiety, Crystal structure elucidation 


\section{Introduction}

Enone system of chalcones is almost planar with trans-double bond. This structure enables various transformations of enone system, which undergo cyclization reactions with urea, thiourea, hydroxylamine, hydrazine, guanidine, $\underline{12}$ forming heterocyclic unit between aromates, and active methylenic compounds (malononitrile, esters of cyanoacetic and acetoacetic acid, acetylacetone, nitromethane $\underline{34}$ Sole double bond of enone system is reactive toward Michael initiated ring closure (MIRC) as well as the sulfoxonium salts (CoreyChaykovsky reaction), $\underline{5}$ yielding cyclopropane derivatives.

A pungent constituent of ginger rhizome dehydrozingerone (1; 4-(4-hydroxy-3-methoxyphenyl)-3-buten-2-one), as a half analogue of curcumin, is present in different bioactive compounds, showing broad spectrum of biological activities, such as anti-inflammatory, antidepressant, antibacterial, antiviral, anticancer $\underline{6}-\underline{12}$ and many others. Although conjugate enone system is presented in this phenolic compound, its structure differs from chalcones in possessing the methyl group connected to carbonyl instead of the aryl one. This unique chalcone-like structure, planar enone system and aromatic ring offer bifunctional site for various transformations. 
As cyclopropane ring is present in a huge number of molecules isolated from nature, $\underline{13}$ such as terpenes, fatty acids, alkaloids and steroids so it is no surprise that many of them show pronounced biological activities, from enzyme inhibition of herpes roteases $\underline{1415}$ to antibiotic, herbicidal, antitumor, and antiviral properties.16-18 Also, well-known are chrysanthemic acid, pyrethrin, and pyrethroid derivatives, as compounds related to natural and synthetic insecticides, with good insecticidal activities. 1920 This motif is attached to main frame of the molecules at different ways. Cyclopropyl group is connected to $\mathrm{C}$-atom in heterocyclic fragment $\underline{21}$ or nitrogen atom in ciprofloxacin derivatives, $\underline{22}$ to carbon chain of $\mathrm{C}_{29}$ sterols, 23 as 1,1-dichloro derivatives of diaryl cyclopropanes.2425 It is also present in combretastatin derivatives as cyclopropyl-vinyl or a cyclopropyl-amide bridge, 2627 in cyclopropyl indolequinones $\underline{28}$ or cinnamic acid derivatives. $\underline{29}$

Based on data from different sources, nearly $50 \%$ of all modern scientific drugs on the market are natural products or natural-based materials and they play an important role in a drug design in pharmaceutical industries. 30 Although many drugs have been introduced into the market, their response to therapy is still poor. For this reasons more efficient drugs should be developed.

Radiotherapy and chemotherapy, as the most frequently used procedures in cancer treatment, are not specific to cancer cells and are known to cause severe and often adverse side effects, such as gastrointestinal reactions, immune suppression, bone marrow 
suppression, hair loss, nerve injury, and even development of secondary malignancies. Therefore, we decided to modify dehydrozingerone, as a natural product easily accessible by simple synthetic procedure, into a new group of compounds. In this way attractive motif, cyclopropyl ring was introduced into the molecule. Microbiological and cytotoxic properties of synthesized compounds had been tested.

\section{Results and Discussion}

\section{Chemistry}

Due to our research interest for the synthesis of molecules exhibiting some biological activity, 1231-34 herein we described the synthesis and characterization, starting from dehydrozingerone (4-(4-hydroxy-3-methoxyphenyl)-3-buten-2-one; 1), of a series of compounds $\mathbf{2 a}-\mathbf{2 j}$. In these compounds, the trans-olefinic bond is replaced by a conformationally restricted and inherent ring strain cyclopropyl moiety, affording a series of novel cyclopropyl derivatives $\mathbf{3 a}-\mathbf{3 j}$.

Dehydrozingerone was synthesized following previously described procedure. $\underline{35}$ Alkylation of their free phenolic group was performed in the boiling acetone solution in the presence of corresponding alkyl halide and anh. $\mathrm{K}_{2} \mathrm{CO}_{3}$, yielding alkyl derivatives, 4-(4-alkoxy-3-methoxyphenyl)-3-buten-2-ones $\mathbf{2 a}-\mathbf{2 j}$. Isolated alkyl derivatives were used 
as substrates in Corey-Chaykovsky reaction. Knowing that this reaction is stereospecific, the series of trans-cyclopropyl products were synthesized (Scheme 1). The prepared compounds, 1-acetyl-2-(4-alkoxy-3-methoxyphenyl)cyclopropanes 3, were characterized by their spectral

data (IR, MS, ${ }^{1} \mathrm{H}-$ and ${ }^{13} \mathrm{C}$-NMR). All four protons from the cyclopropane ring have a different environment, and each of them is coupling with others and four groups of signals are presented. As result of it, each proton has three different coupling constants. The crystal structure of 1-acetyl-2-(4-isopropoxy-3-methoxyphenyl)-cyclopropane (3c) was also reported.

\section{X-Ray Analysis}

The crystal structure of molecule $\mathbf{3 c}$ and the selected geometrical parameters are given in Fig. 1, while the selected geometrical parameters are given in Table 1 . The $\mathrm{C}(3)-\mathrm{C}(5)$ bond of the middle cyclopropane fragment $(\mathrm{cp})$ is the longest bond in the structure. A slight elongation of this bond in comparison to the other two $\mathrm{C}-\mathrm{C}$ bonds of the strained $\mathrm{cp}$ ring can be attributed to the presence of the acetyl and aryl substituents at the $\mathrm{C}(3)$ and $\mathrm{C}(5)$ atoms, respectively. 


$$
\begin{aligned}
& \text { a) } \\
& \text {, } \\
& 1
\end{aligned}
$$

Sheme 1. Synthesis of cyclopropyl derivates 3-a-3j of dehydrozingerone a) acetone. $\mathrm{K}_{2} \mathrm{CO}_{3}, \mathrm{RX}$, reflux overnight; $b$ ) dry DMSO, $\mathrm{NaH},\left.\mathrm{Me}_{3} \mathrm{SO}^{+}\right|^{-}$

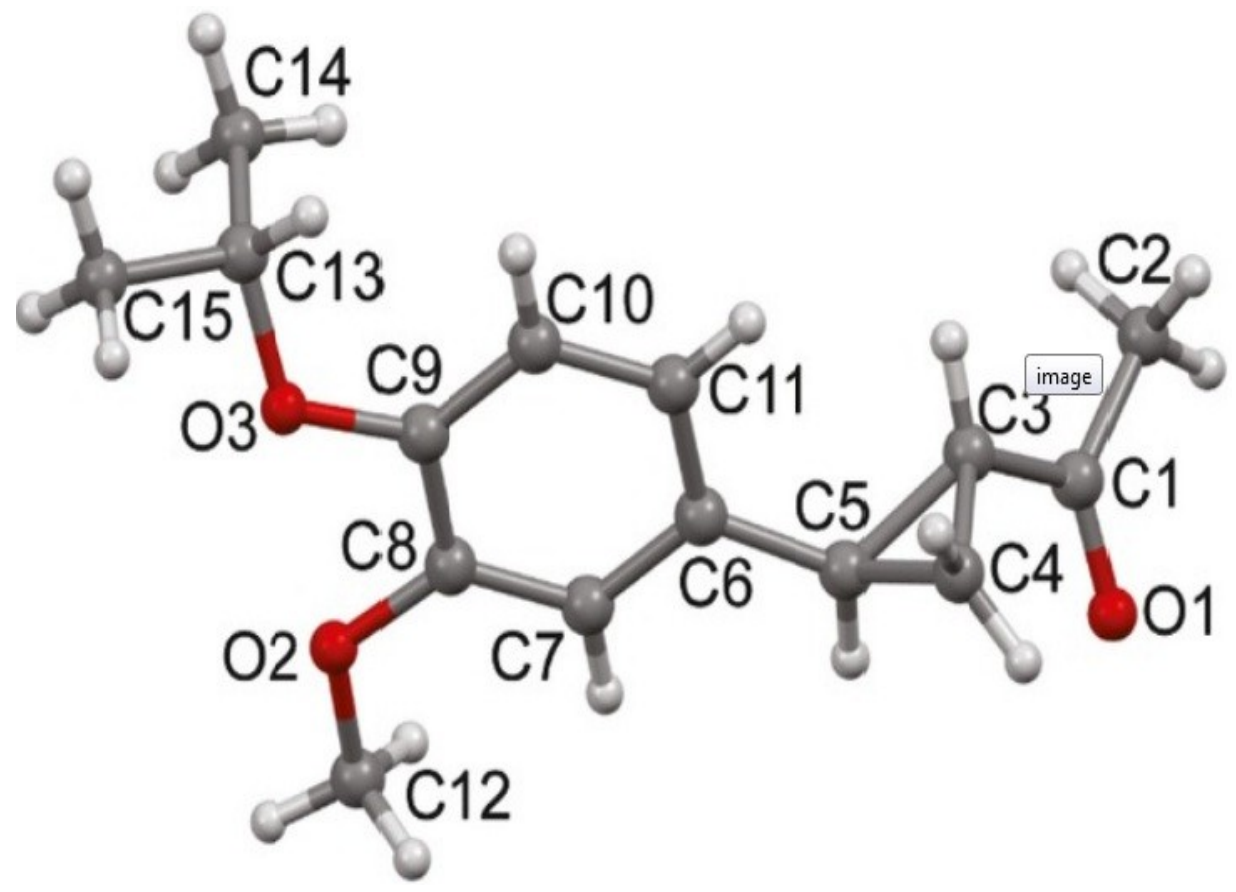

Figure 1 Crystal structure of compound $\mathbf{3 c}$. 
Table 1. Selected bond lenghts $[\AA ̊]$ and angles $\left[{ }^{\circ}\right]$ of compound $\mathbf{3 c}$

\begin{tabular}{|c|c|c|c|}
\hline & Bond length & & Angle \\
\hline$C(1)-O(1)$ & $1.212(2)$ & $C(2)-C(1)-O(1)$ & $121.5(2)$ \\
\hline $\mathrm{C}(8)-\mathrm{O}(2)$ & $1.369(2)$ & $C(3)-C(1)-O(1)$ & $121.2(2)$ \\
\hline $\mathrm{C}(9)-\mathrm{O}(3)$ & $1.372(2)$ & $C(8)-O(2)-C(12)$ & $117.6(1)$ \\
\hline$C(3)-C(4)$ & $1.493(3)$ & $C(9)-O(3)-C(13)$ & $119.0(1)$ \\
\hline$C(4)-C(5)$ & $1.485(2)$ & $C(3)-C(4)-C(5)$ & $61.6(1)$ \\
\hline \multirow[t]{2}{*}{$C(3)-C(5)$} & $1.524(2)$ & $C(4)-C(5)-C(3)$ & $59.5(1)$ \\
\hline & & $C(5)-C(3)-C(4)$ & $59.0(1)$ \\
\hline
\end{tabular}

The angle opposite to the $\mathrm{C}(3)-\mathrm{C}(5)$ bond also slightly expands at the expense of the remaining two angles (Table 1 ). Similar asymmetry within the disubstituted $\mathrm{cp}$ ring has been observed in related crystal structures such as phenylcyclopropanecarboxylic acids. $\underline{36}-\underline{38}$ The angles outside the cp ring depend on the size of the attached substituents; thus, the angles between the $\mathrm{C}-\mathrm{C}$ bonds of the cp ring and the corresponding bonds attaching the aryl and acetyl fragments have the average values of $121.3^{\circ}$ and $117.6^{\circ}$, respectively.

The dihedral angle between the phenyl and cp rings of $87.8(1)^{\circ}$ describes the nearly perpendicular arrangement of these two systems, as also observed in the case of the free 
phenylcyclopropane $\left(88.6^{\circ}\right) .39$ In addition, the best plane of the phenyl ring in $\mathbf{3 c}$ bisects the opposite cp $\mathrm{C}(3)-\mathrm{C}(4)$ bond so the torsion angle $\mathrm{C}(11)-\mathrm{C}(6)-\mathrm{C}(5)-\mathrm{Ct} 1$ (where $\mathrm{Ct} 1$ is the midpoint of $\mathrm{C}(3)-\mathrm{C}(4))$ equals to $6.1^{\circ}$. Acetyl fragment displays similar perpendicular position with respect to the middle cp ring with the dihedral angle between the best planes $\mathrm{C}(2) / \mathrm{C}(1) / \mathrm{O}(1)$ and $\mathrm{C}(3) / \mathrm{C}(4) / \mathrm{C}(5)$ of $88.9(1)^{\circ}$ and the torsion angle $\mathrm{O}(1)-\mathrm{C}(1)-\mathrm{C}(3)-\mathrm{Ct} 2$ of $1.52^{\circ}$ (where $\mathrm{Ct} 2$ is the midpoint of $\mathrm{C}(4)-\mathrm{C}(5)$ ). The mutual orientation of the acetyl and phenyl systems is described by the torsion angle $C(1)-C(3)-C(5)-C(6)$ which equals to $-143.5(1)^{\circ}$. Similarly to the previously described methoxyphenyl derivatives, $\underline{12}$ the methoxy fragment attached to the phenyl ring only slightly deviates from the phenyl mean plane $(\mathrm{O}(2)$ : -0.019 (2); $C(12):-0.077$ (3) $\AA$ ); this is in contrast to the i-propoxy moiety where the deviation of the corresponding atoms is more pronounced $(\mathrm{O}(3)$ : $0.078(2) ; \mathrm{C}(13)$ : -0.308 (3) A).

In the crystal, the centrosymmetrically related molecules form dimers by a pair of

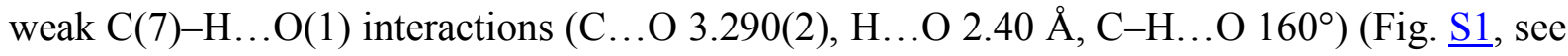
Supporting Information). Further arrangement of the adjacent molecules is based on weak $\mathrm{C}(4)-\mathrm{H}(4 \mathrm{~b}) \ldots \pi$ interaction $(\mathrm{C} \ldots \mathrm{Cg} 13.464(2), \mathrm{H} \ldots \pi 2.78 \AA ⿻$, C-H...Cg1 129, where $\mathrm{Cg} 1$ is the centroid of phenyl ring) (Fig. S2). 


\section{Antimicrobial Activity}

The antimicrobial activities of the investigated compounds against the test microorganisms are shown in Tables $\underline{2}$ and $\underline{3}$. The tested compounds demonstrated relatively strong antimicrobial activity inhibiting all tested microorganisms. The minimum inhibitory concentration $(M I C)$ for these compounds relative to the tested bacteria ranged from 0.009 to

$1.25 \mathrm{mg} / \mathrm{ml}$ (Table 2). The strongest antibacterial activity was found in $\mathbf{3 b}$ compound inhibiting all the species of bacteria in low concentrations, especially Bacillus subtilis where measured $M I C$ value was $0.009 \mathrm{mg} / \mathrm{ml}$ (stronger than streptomycin). Among the bacteria, Escherichia coli showed the highest resistance while $B$. subtilis was the most sensitive. The tested compound also inhibited the growth of the all tested fungi (Table $\underline{3}$ ) but in slightly higher concentrations (MIC values were from 0.156 to $5 \mathrm{mg} / \mathrm{ml}$ ). Among the fungi, Candida albicans appeared to be the most sensitive.

The antimicrobial activity was compared with the standard antibiotics, streptomycin (for bacteria) and ketoconazole (for fungi). The experimental results showed that the tested compound had just slightly weaker effect than streptomycin (compound $\mathbf{3 b}$ even better than streptomycin), while ketoconazole had stronger activity than the tested samples as shown in Tables $\underline{2}$ and $\underline{3}$. In a negative control, DMSO had no inhibitory effect on the tested organisms.

In these experiments, antibacterial effect was observed against the both Gram-positive and Gram-negative bacteria but it should be noted that the Gram-negative bacteria were more 
resilient. It has been generally reported that the Gram-negative bacteria are more resistant than Gram-positive. ${ }^{[12][40][41]}$ This resistance is likely, due to the fact that Gram-negative bacteria have a wall which itself is surrounded by an outer complex membrane, slowing down the passage of hydrophobic compounds.

Table 2. Antimicrobial activities of tested compounds

\begin{tabular}{|c|c|c|c|c|c|}
\hline Microorganisms & Staphylococcus aureus & Bacillus subtilis & Bacillus cereus & Escherichia coli & Proteus mirabilis \\
\hline $3 a$ & 0.312 & 0.019 & 0.078 & 0.312 & 0.312 \\
\hline $3 \mathbf{b}$ & 0.078 & 0.009 & 0.019 & 0.312 & 0.156 \\
\hline 3c & 0.312 & 0.019 & 0.078 & 1.25 & 0.625 \\
\hline 3d & 0.156 & 0.019 & 0.039 & 0.625 & 0.312 \\
\hline $3 e$ & 0.156 & 0.078 & 0.078 & 0.625 & 0.312 \\
\hline $3 \mathbf{f}$ & 0.312 & 0.019 & 0.019 & 1.25 & 0.312 \\
\hline $3 g$ & 0.156 & 0.078 & 0.078 & 0.312 & 0.156 \\
\hline $3 \mathrm{~h}$ & 0.625 & 0.039 & 0.078 & 1.25 & 1.25 \\
\hline $3 \mathbf{i}$ & 0.156 & 0.039 & 0.078 & 0.312 & 0.156 \\
\hline $3 \mathbf{j}$ & 0.156 & 0.078 & 0.078 & 0.625 & 0.312 \\
\hline Streptomycin & 0.031 & 0.016 & 0.016 & 0.062 & 0.062 \\
\hline
\end{tabular}

Minimum inhibitory concentration given as $\mathrm{mg} / \mathrm{ml}$. The values are the mean of three replicate. In all cases the standard deviation is \pm 0.002 . 
Table 3 Antimicrobial activities of tested compounds.

\begin{tabular}{|c|c|c|c|c|c|}
\hline Microorganisms & Aspergillus flavus & Aspergillus fumigatus & Candida albicans & Penicillium purpurescens & Penicillium verucosum \\
\hline $3 a$ & 1.25 & 0.312 & 0.625 & 0.625 & 0.625 \\
\hline $3 b$ & 0.625 & 0.312 & 0.312 & 0.312 & 0.625 \\
\hline $3 c$ & 1.25 & 1.25 & 0.625 & 2.5 & 2.5 \\
\hline 3d & 0.625 & 0.312 & 0.625 & 0.312 & 0.625 \\
\hline 3e & 5 & 1.25 & 0.156 & 2.5 & 2.5 \\
\hline $3 f$ & 1.25 & 0.625 & 0.625 & 1.25 & 2.5 \\
\hline $3 g$ & 2.5 & 0.625 & 0.312 & 1.25 & 2.5 \\
\hline $3 h$ & 2.5 & 1.25 & 1.25 & 2.5 & 2.5 \\
\hline $3 \mathbf{i}$ & 2.5 & 0.625 & 0.312 & 1.25 & 2.5 \\
\hline $3 \mathbf{j}$ & 2.5 & 1.25 & 0.156 & 2.5 & 1.25 \\
\hline Ketoconazole & 0.156 & 0.078 & 0.039 & 0.156 & 0.156 \\
\hline
\end{tabular}

Minimum inhibitory concentration given as $\mathrm{mg} / \mathrm{ml}$. The values are the mean of three replicate. In all cases the standard deviation is \pm 0.001 .

Lacking outer membrane, Gram-positive bacteria are more susceptible to the antibiotic agents. ${ }^{[42]}$ Compared to the bacteria, fungi were more resistant due to the more complex structure of the cell wall ${ }^{[43]}$ 


\section{Cytotoxic Activity}

The cytotoxicity of cyclopropyl derivatives $\mathbf{3 a}-\mathbf{3} \mathbf{j}$ against the human carcinoma cell lines HeLa, A549, and LS174 was assessed by the MTT test, using cis-DDP as the control. The cytotoxic activity of tested compounds is given in Table $\underline{4}$, as the $I C_{50}$ values $[\mu \mathrm{m}] . I C_{50}$ values are expressed as the mean \pm SD determined from the results of MTT assay in three independent experiments.

As noted in the table, all samples showed marked cytotoxic activity against tested carcinoma cell lines. Compound $\mathbf{3 h}$ showed the highest effect against HeLa cells $\left(I C_{50}=8.63\right.$ $\mu \mathrm{m})$, whereas compound $\mathbf{3 f}$ showed best activity against LS174 and A549 cell lines $\left(I C_{50}=\right.$ 10.17 and $12.15 \mu \mathrm{m}$, respectively).

Moreover, compared to $c i s$-DDP, all samples showed no cytotoxicity toward normal tissue cells, human fetal lung fibroblast cell line (MRC5) $\left(I C_{50}>200\right)$. Selectivity index, defined as $I C_{50}(\mathrm{MRC} 5) / I C_{50}$ (cell line) ratio (Table ), indicates that all tested compounds exhibit superior selectivity comparing to cis-DDP (up to ten times). According to the supreme cytotoxicity and selectivity (low $I C_{50}$ and high selectivity index), compounds $\mathbf{3 e}, \mathbf{3 f}$, and $\mathbf{3 h}$ demonstrate the most promising cytotoxic activity against the selected carcinoma cell lines. 
Table 4. Cytotoxic activity ( $I C_{50}$ values) of compounds $3 \mathrm{a}-3 \mathrm{j}$ against human cancer cell lines HeLa, LS174, A549, and human fetal lung fibroblast cell line

(MRC5)

\begin{tabular}{|c|c|c|c|c|}
\hline \multirow[t]{2}{*}{ Compounds } & \multicolumn{4}{|l|}{$I_{50}[\mu \mathrm{M}]$} \\
\hline & HeLa & LS174 & A549 & MRC5 \\
\hline $3 a$ & $38.34 \pm 1.95$ & $40.91 \pm 1.22$ & $24.05 \pm 2.43$ & $>200$ \\
\hline $\mathbf{3 b}$ & $24.14 \pm 0.76$ & $29.76 \pm 0.57$ & $32.98 \pm 0.72$ & $>200$ \\
\hline $3 c$ & $16.22 \pm 1.48$ & $36.36 \pm 2.72$ & $28.77 \pm 3.41$ & $>200$ \\
\hline 3d & $38.86 \pm 2.59$ & $41.57 \pm 0.79$ & $36.37 \pm 2.55$ & $>200$ \\
\hline $3 e$ & $17.61 \pm 1.87$ & $18.73 \pm 0.55$ & $16.85 \pm 1.32$ & $>200$ \\
\hline $3 f$ & $34.63 \pm 2.81$ & $10.17 \pm 1.23$ & $12.15 \pm 0.49$ & $>200$ \\
\hline $3 g$ & $12.16 \pm 1.84$ & $31.11 \pm 2.59$ & $30.85 \pm 1.20$ & $>200$ \\
\hline $3 h$ & $8.63 \pm 0.49$ & $17.53 \pm 2.45$ & $26.89 \pm 0.77$ & $>200$ \\
\hline $3 \mathbf{i}$ & $19.18 \pm 0.62$ & $23.74 \pm 1.36$ & $26.63 \pm 3.48$ & $>200$ \\
\hline $3 \mathbf{j}$ & $24.52 \pm 1.14$ & $32.52 \pm 1.81$ & $32.11 \pm 0.31$ & $>200$ \\
\hline cis-DDP & $2.45 \pm 0.21$ & $5.03 \pm 0.47$ & $9.48 \pm 0.73$ & $15.22 \pm 0.68$ \\
\hline
\end{tabular}

'Bold' type refers to the most active compound on corresponding cell line. 
Tabele 5. Selectivity index

\begin{tabular}{|c|c|c|c|}
\hline \multirow[t]{2}{*}{ Compounds } & HeLa & LS174 & A549 \\
\hline & \multicolumn{3}{|c|}{$/ C_{50}(\mathrm{MRC5}) / / C_{50}(\mathrm{cell}$ line) } \\
\hline $3 a$ & 5.2 & 4.9 & 8.3 \\
\hline $3 b$ & 8.3 & 6.7 & 6.1 \\
\hline $3 c$ & 12.3 & 5.5 & 7.0 \\
\hline $3 d$ & 5.1 & 4.8 & 5.5 \\
\hline $3 e$ & 11.4 & 10.7 & 11.9 \\
\hline $3 f$ & 5.8 & 19.7 & 16.5 \\
\hline $3 g$ & 16.4 & 6.4 & 6.5 \\
\hline $3 \mathrm{~h}$ & 23.2 & 11.4 & 7.4 \\
\hline $3 \mathbf{i}$ & 10.4 & 8.4 & 7.5 \\
\hline $3 j$ & 8.2 & 6.2 & 6.2 \\
\hline cis-DDP & 6.2 & 3.0 & 1.6 \\
\hline
\end{tabular}




\section{Conclusions}

Dehydrozingerone, as an easily accessible natural product, was modified by the simple synthetic procedure. In the present study, the results clearly demonstrate that, although simple by structure, the synthesized compounds show significant microbiological activities toward tested strains of microorganisms; the strongest activity was observed towards Bacillus strains of bacteria. Moreover, in vitro cytotoxic activity of the synthesized compounds against tumor cell lines, human epithelial carcinoma HeLa cells, human colon carcinoma LS174 cells and human lung carcinoma A549 cells was observed. Most importantly, compared to cis-DDP, the synthesized compounds showed very low cytotoxicity $\left(I C_{50}>200\right)$ toward normal human fetal lung fibroblast MRC5 cell line.

The good activity of synthesized compounds, fewer side effects, along with the lack of toxicity (very low cytotoxicity against MRC5 cells) and the feasible synthesis, underscore their value as promising novel scaffolds for the development of new antimicrobial and anticancer drugs.

\section{Experimental Section}

\section{Chemistry}

All starting chemicals were commercially available and used as received, except for the solvents being purified by distillation. (E)-4-(4-Hydroxy-3-methoxyphenyl)but-3-en-2-one, 
dehydrozingerone (1), was prepared following the previously mentioned procedure. $\underline{35}$ $O$-Alkyl dehydrozingerones were synthesized following described procedures. $\underline{44}-\underline{46}$ The chemical synthesis of compounds $\mathbf{2 a}-\mathbf{2 d}$ and $\mathbf{2 f}-\mathbf{2 i}$ was published earlier. 47 Isobutyl derivative $\mathbf{2 e}$ and methallyl derivative $\mathbf{2} \mathbf{j}$ are new compounds and their structure and spectral data are already given. Out of the products of type $\mathbf{3}$, only compound 3a was earlier described, $\underline{48}$ whereas $\mathbf{3 b}-\mathbf{3} \mathbf{j}$ are new compounds, prepared according described procedure $\underline{5}$ (Scheme 1).

Column chromatography: silica gel 60 (Merck, 230 - 400 mesh ASTM). TLC: Silica gel 60 F254-precoated plates (Merck). IR Spectra: PerkinElmer Spectrum One FT-IR spectrometer with a $\mathrm{KBr}$ disc, $v$ in $\mathrm{cm}^{1}$. NMR Spectra: Varian Gemini $200 \mathrm{MHz}$ spectrometer $(200 \mathrm{MHz}$ for ${ }^{1} \mathrm{H}$ and $50 \mathrm{MHz}$ for ${ }^{13} \mathrm{C}$ ), using $\mathrm{CDCl}_{3}$ as the solvent and TMS as the internal standard. ${ }^{1} \mathrm{H}$ and ${ }^{13} \mathrm{C}$-NMR chemical shifts were reported in parts per million [ppm] and were referenced to the solvent peak; $\mathrm{CDCl}_{3}$ (7.26 ppm for ${ }^{1} \mathrm{H}$ and $76.90 \mathrm{ppm}$ for $\left.{ }^{13} \mathrm{C}\right)$. Multiplicities are represented by $s$ (singlet), $d$ (doublet), $t$ (triplet), $q$ (quartet), ddd (doublet of doublet of doublet), and $m$ (multiplet). Coupling constants $(J)$ are in Hertz [Hz]. Mass spectrometry was performed by Waters Micromass ZQ mass spectrometer and MassLynx software for control and data processing. Electro spray ionization in the positive mode was used. The electro spray capillary was set at $4.3 \mathrm{kV}$ and the cone at $40 \mathrm{~V}$. The ion source temp. was set at $125^{\circ} \mathrm{C}$ and the nitrogen flow rates were 400 and $50 \mathrm{l} / \mathrm{h}$, for desolvation and cone gas flow, resp. The 
collision energy was $40 \mathrm{eV}$. The melting point of products was determined by using MelTemp1000 apparatus.

General Procedure for the Synthesis of 4-(4-Alkoxy-3-methoxyphenyl)-but-3-en-2-ones (2a$2 j$. )A mixture of dehydrozingerone (1;1.92 g, $10 \mathrm{mmol}$ ), appropriate alkyl halide (excess, 30 mmol) and $\mathrm{K}_{2} \mathrm{CO}_{3}(7 \mathrm{~g}$, anh.) in acetone $(60 \mathrm{ml})$ was heated to reflux overnight under argon. Acetone and the excess of alkyl halide were evaporated under reduced pressure and the solid residue was dissolved in water. The mixture was distilled with steam to remove excess of alkyl halide and their side products. After cooling, the water mixture was extracted with $\mathrm{CH}_{2} \mathrm{Cl}_{2}(3 \times 50 \mathrm{ml})$. The combined extracts were washed with water and dried over anh. $\mathrm{Na}_{2} \mathrm{SO}_{4}$. After the removal of the main part of solvent, the residue was filtered over silica gel pad. Compounds $2 \mathrm{e}$ and $2 \mathrm{j}$ were isolated as oils, while others were isolated as white crystalline substances.

(3E)-4-[3-Methoxy-4-(2-methylpropoxy)phenyl]-but-3-en-2-one (2e). Yield: $66 \%$. IR (KBr): 2956, 1665, 1583, 1518, 1422, 1255, 1138, 1022. ${ }^{1} \mathrm{H}-\mathrm{NMR}\left(200 \mathrm{MHz}, \mathrm{CDCl}_{3}\right): 1.06$ $(d, J=6.6,6 \mathrm{H}) ; 2.05-2.25(m, 1 \mathrm{H}) ; 2.37(s, 3 \mathrm{H}) ; 3.81(d, J=6.8,2 \mathrm{H}) ; 3.89(s, 3 \mathrm{H}) ; 6.59$ $(d, J=16.2,1 \mathrm{H}) ; 6.84-7.12(m, 3 \mathrm{H}) ; 7.46(d, J=16.2,1 \mathrm{H}) .{ }^{13} \mathrm{C}-\mathrm{NMR}\left(50 \mathrm{MHz}, \mathrm{CDCl}_{3}\right)$ : $19.2 ; 27.2 ; 28.1 ; 56.1 ; 75.4 ; 110.6 ; 112.7 ; 122.9 ; 125.1 ; 127.1 ; 143.6 ; 149.7 ; 151.3 ; 198.3$ (CO). 
(3E)-4-\{3-Methoxy-4-[(2-methylprop-2-en-1-yl)oxy]phenyl\}but-3-en-2-one (2j). Yield: 62\%. IR (KBr): 2979, 1665, 1641, 1593, 1517, 1424, 1263, 1168, 1144, 1032, 980. ${ }^{1} \mathrm{H}-\mathrm{NMR}$ (200 MHz, $\left.\mathrm{CDCl}_{3}\right): 1.83(s, 3 \mathrm{H}) ; 2.37(s, 3 \mathrm{H}) ; 3.91(s, 3 \mathrm{H}) ; 4.56(s, 2 \mathrm{H}) ; 5.00-5.09(m, 2$ $\mathrm{H}) ; 6.59(d, J=16.2,1 \mathrm{H}) ; 6.86(d, J=8.8,1 \mathrm{H}) ; 7.05-7.11(m, 2 \mathrm{H}) ; 7.45(d, J=16.4,1 \mathrm{H})$. ${ }^{13} \mathrm{C}-\mathrm{NMR}\left(50 \mathrm{MHz}, \mathrm{CDCl}_{3}\right): 19.2 ; 27.2 ; 55.9 ; 72.5 ; 110.3 ; 112.9 ; 113.1 ; 122.7 ; 125.2 ; 127.5$; $140.2 ; 143.4 ; 149.7 ; 150.6 ; 198.1(\mathrm{CO})$.

General Procedure for the Synthesis of 1-Acetyl-2-(4-alkoxy-3-methoxyphenyl)cyclopropanes $(3 a-3 j)$. The solution of the ylide was prepared under nitrogen from sodium hydride (3 mmol, $0.15 \mathrm{~g}$ of $50 \%$ oil suspension), trimethyloxosulfonium iodide $(0.726 \mathrm{~g}, 3.3 \mathrm{mmol})$ and DMSO (3 ml). Enone solution in DMSO (3 mmol in $5 \mathrm{ml}$ ) was added to this mixture while being stirred and cooled in a water bath. The mixture was stirred at r.t. for $2 \mathrm{~h}$ and then at 50 ${ }^{\circ} \mathrm{C}$ for $1 \mathrm{~h}$. The solvent mixture of toluene/AcOEt $(95: 5,20 \mathrm{ml})$ was added to a reaction flask with stirring. The mixture was then poured into $50 \mathrm{ml}$ of cold water and extracted with toluene. The extracts were washed twice with water, dried over anh. $\mathrm{Na}_{2} \mathrm{SO}_{4}$ and evaporated, yielding pale yellow oil. The crude mixture was chromatographed on silica gel column, using hexane/AcOEt mixture (7:3).

1-[2-(3,4-Dimethoxyphenyl)cyclopropyl]ethanone (3a). Yield: $68 \%$. M.p. $63-64{ }^{\circ} \mathrm{C}$. IR (KBr): 3001, 2835, 1695, 1608, 1590, 1519, 1397, 1258, 1236, 1141, 1028, 968. ${ }^{1} \mathrm{H}-\mathrm{NMR}$ $\left(200 \mathrm{MHz}, \mathrm{CDCl}_{3}\right): 1.35(d d d, J=4.2,6.8,8.2,1 \mathrm{H}) ; 1.64(d d d, J=4.2,5.2,9.2,1 \mathrm{H}) ; 2.17$ 
$(d d d, J=4.0,5.2,8.2,1 \mathrm{H}) ; 2.30(s, 3 \mathrm{H}) ; 2.49(d d d, J=4.0,6.8,9.0,1 \mathrm{H}) ; 3.85(s, 3 \mathrm{H}) ; 3.87$ $(s, 3 \mathrm{H}) ; 6.62-6.66(m, 2 \mathrm{H}) ; 6.79(d, J=8.6,1 \mathrm{H}) .{ }^{13} \mathrm{C}-\mathrm{NMR}\left(50 \mathrm{MHz}, \mathrm{CDCl}_{3}\right): 18.7 ; 28.7$; 30.6; 32.6; 55.8; 55.9; 109.9; 111.3; 117.8; 132.7; 147.8; 148.9; 206.6 (CO). ESI-MS (40 eV): 220 (14), 177 (42), 164 (10), 151 (15.5), 138 (8), 91 (61).

1-[2-(4-Ethoxy-3-methoxyphenyl)cyclopropyl]-ethanone (3b). Yield: 69\%. M.p. 65 - 66 ${ }^{\circ} \mathrm{C}$. IR (KBr): 3004, 2933, 1689, 1609, 1589, 1520, 1399, 1266, 1231, 1140, 1037, 965, 846. ${ }^{1} \mathrm{H}-\mathrm{NMR}\left(200 \mathrm{MHz}, \mathrm{CDCl}_{3}\right): 1.35(d d d, J=4.0,6.8,8.2,1 \mathrm{H}) ; 1.44(t, J=7.0,3 \mathrm{H}) ; 1.63$ $(d d d, J=4.0,5.0,9.0,1 \mathrm{H}) ; 2.16(d d d, J=4.0,5.0,8.2,1 \mathrm{H}) ; 2.30(s, 3 \mathrm{H}) ; 2.48(d d d, J=4.0$, $6.8,9.0,1 \mathrm{H}) ; 3.86(s, 3 \mathrm{H}) ; 4.07(q, J=7.0,2 \mathrm{H}) ; 6.58-6.63(m, 2 \mathrm{H}) ; 6.79(d, J=8.0,1 \mathrm{H})$. ${ }^{13} \mathrm{C}-\mathrm{NMR}\left(50 \mathrm{MHz}, \mathrm{CDCl}_{3}\right): 14.7 ; 18.8 ; 28.8 ; 30.7 ; 32.7 ; 55.8 ; 64.3 ; 110.1 ; 112.8 ; 117.8$; 132.7; 147; 149.2; 206.8 (CO). ESI-MS (40 eV): 234 (17), 191 (33), 189 (26), 165 (28).

1-\{2-[3-Methoxy-4-(propan-2-yloxy)phenyl]cyclopropyl\}ethanone (3c). Yield: 70\%. Oil. IR (KBr): 2976, 2935, 1697, 1607, 1586, 1515, 1397, 1385, 1259, 1230, 1139, 1112, 1038, 848. ${ }^{1} \mathrm{H}-\mathrm{NMR}\left(200 \mathrm{MHz}, \mathrm{CDCl}_{3}\right): 1.35(d d d, J=4.2,6.8,8.4,1 \mathrm{H}) ; 1.33(d, J=6.2,6 \mathrm{H})$; $1.64(d d d, J=4.4,5.4,9.4,1 \mathrm{H}) ; 2.18 .(d d d, J=4.2,5.2,8.2,1 \mathrm{H}) ; 2.30(s, 3 \mathrm{H}) ; 2.48(d d d, J$ $=4.2,6.8,9.0,1 \mathrm{H}) ; 3.84(s, 3 \mathrm{H}) ; 4.41-4.53(m, 1 \mathrm{H}) ; 6.57-6.65(m, 2 \mathrm{H}) ; 6.81(d, J=8.0$, $1 \mathrm{H}) .{ }^{13} \mathrm{C}-\mathrm{NMR}\left(50 \mathrm{MHz}, \mathrm{CDCl}_{3}\right): 18.8 ; 22 ; 28.8 ; 30.6 ; 32.7 ; 55.9 ; 71.6 ; 110.6 ; 116.4 ; 117.8$; 133.3; 146; 150.5; 206.7 (CO). ESI-MS (40 eV): 248 (17), 206 (81), 189 (100), 163 (68), 157 (26), 137 (24). 
1-[2-(3-Methoxy-4-propoxyphenyl)cyclopropyl]-ethanone (3d). Yield: 79\%. M.p. 48 - 49 ${ }^{\circ} \mathrm{C}$. IR (KBr): 2967, 2940, 1683, 1610, 1588, 1521, 1389, 1260, 1237, 1136, 1017, 975, 843. ${ }^{1} \mathrm{H}-\mathrm{NMR}\left(200 \mathrm{MHz}, \mathrm{CDCl}_{3}\right): 1.03(t, J=7.6,3 \mathrm{H}) ; 1.33(d d d, J=4.4,6.6,8.2,1 \mathrm{H}) ; 1.64$ $(d d d, J=4.0,5.2,9.2,1 \mathrm{H}) ; 1.76-1.92(m, 2 \mathrm{H}) ; 2.17(d d d, J=4.0,5.2,8.2,1 \mathrm{H}) ; 2.30(s, 3$ $\mathrm{H}) ; 2.48(d d d, J=4.0,6.6,9.2,1 \mathrm{H}) ; 3.85(s, 3 \mathrm{H}) ; 3.95(t, J=6.8,2 \mathrm{H}) ; 6.58-6.64(m, 2 \mathrm{H})$; $6.79(d, J=8.0,1 \mathrm{H}) .{ }^{13} \mathrm{C}-\mathrm{NMR}\left(50 \mathrm{MHz}, \mathrm{CDCl}_{3}\right): 10.3 ; 18.7 ; 22.4 ; 28.8 ; 30.6 ; 32.7 ; 55.9$; 70.6; 110.4; 113.2; 117.9; 132.7; 147.3; 149.4; 206.7 (CO). ESI-MS (40 eV): 248 (13), 205 (53), $189(48), 163(33)$.

1-\{2-[3-Methoxy-4-(2-methylpropoxy)phenyl]cyclopropyl\}ethanone $\quad$ (3e). Yield: $53 \%$. M.p. $50-51^{\circ} \mathrm{C}$. IR (KBr): 2959, 2906, 1684, 1588, 1522, 1389, 1258, 1234, 1025, 976, 844. ${ }^{1} \mathrm{H}-\mathrm{NMR}\left(200 \mathrm{MHz}, \mathrm{CDCl}_{3}\right): 1.02(d, J=6.6,6 \mathrm{H}) ; 1.34(d d d, J=4.4,6.8,8.2,1 \mathrm{H}) ; 1.64$ $(d d d, J=4.4,5.2,9.2,1 \mathrm{H}) ; 2.14(d d d, J=4.0,5.2,8.2,1 \mathrm{H}) ; 2.30(s, 3 \mathrm{H}) ; 2.48(d d d, J=4.0$, $6.8,9.2,1 \mathrm{H}) ; 3.74(d, J=7.0,2 \mathrm{H}) ; 3.85(s, 3 \mathrm{H}) ; 6.59-6.63(m, 2 \mathrm{H}) ; 6.79(d, J=8.0,1 \mathrm{H})$. ${ }^{13} \mathrm{C}-\mathrm{NMR}\left(50 \mathrm{MHz}, \mathrm{CDCl}_{3}\right): 18.8 ; 19.2 ; 28.1 ; 28.8 ; 30.7 ; 32.7 ; 56.2 ; 75.8 ; 110.9 ; 113.6 ; 118$; 132.8; 147.8; 149.6; 206.7 (CO). ESI-MS (10 eV): $263\left(100,[M+1]^{+}\right)$.

1-[2-(4-Butoxy-3-methoxyphenyl)cyclopropyl]-ethanone (3f). Yield: 52\%. M.p. 55 - 56 ${ }^{\circ} \mathrm{C} . \mathrm{IR}$ (KBr): 2961, 2872, 1685, 1609, 1589, 1523, 1390, 1259, 1236, 1134, 1033, 1025, 979, 843. ${ }^{1} \mathrm{H}-\mathrm{NMR}\left(200 \mathrm{MHz}, \mathrm{CDCl}_{3}\right): 0.97(t, J=7.4,3 \mathrm{H}) ; 1.32(d d d, J=4.0,6.8,8.2,1 \mathrm{H})$; $1.39-1.54(m, 2 \mathrm{H}) ; 1.63(d d d, J=4.2,5.2,9.2,1 \mathrm{H}) ; 1.74-1.88(m, 2 \mathrm{H}) ; 2.16(d d d, J=$ 
4.2, 5.4, 8.4, $1 \mathrm{H}) ; 2.30(s, 3 \mathrm{H}) ; 2.48(d d d, J=4.0,6.6,9.2,1 \mathrm{H}) ; 3.85(s, 3 \mathrm{H}) ; 3.99(t, J=$ $6.8,2 \mathrm{H}) ; 6.58-6.63(m, 2 \mathrm{H}) ; 6.79(d, J=8.2,1 \mathrm{H}) .{ }^{13} \mathrm{C}-\mathrm{NMR}\left(50 \mathrm{MHz}, \mathrm{CDCl}_{3}\right): 13.8 ; 18.7$; $19.1 ; 28.8 ; 30.7 ; 31.2 ; 32.7 ; 56 ; 68.9 ; 110.5 ; 113.2 ; 117.9 ; 132.7 ; 147.4 ; 148.4 ; 206.7$ (CO). ESI-MS (40 eV): $263\left(11,[M+1]^{+}\right), 219$ (16), 207 (19), 189 (72), 157 (24.5).

1-\{2-[3-Methoxy-4-(3-methylbutoxy)phenyl]cyclopropyl\}ethanone (3g). Yield: 78\%. M.p. $74-75^{\circ} \mathrm{C}$. IR (KBr): 2954, 2868, 1682, 1587, 1521, 1387, 1255, 1237, 1136, 1025, 979, 850. ${ }^{1} \mathrm{H}-\mathrm{NMR}\left(200 \mathrm{MHz}, \mathrm{CDCl}_{3}\right): 0.96(d, J=6.4,6 \mathrm{H}) ; 1.34(d d d, J=4.2,6.8,8.2,1 \mathrm{H}) ; 1.59-$ $1.86(m, 4 \mathrm{H}), 2.16(d d d, J=4.0,5.2,8.2,1 \mathrm{H}) ; 2.30(s, 3 \mathrm{H}) ; 2.48(d d d, J=4.0,6.6,9.0,1$ $\mathrm{H}) ; 3.85(s, 3 \mathrm{H}) ; 4.01(t, J=6.8,2 \mathrm{H}) ; 6.59-6.63(m, 2 \mathrm{H}) ; 6.80(d, J=8.4,1 \mathrm{H}) .{ }^{13} \mathrm{C}-\mathrm{NMR}$ (50 MHz, $\left.\mathrm{CDCl}_{3}\right): 18.8 ; 22.6 ; 25.1 ; 28.8 ; 30.7 ; 32.7 ; 37.9 ; 56.1 ; 67.7 ; 110.5 ; 113.3 ; 117.9$; 132.8; 147.5; 149.5; $206.8(\mathrm{CO})$. ESI-MS (10 eV): $277\left(100,[M+1]^{+}\right)$.

1-\{2-[4-(Benzyloxy)-3-methoxyphenyl]cyclopropyl\}ethanone (3h). Yield: 82\%. M.p. $101-$ $102^{\circ} \mathrm{C}$. IR (KBr): 2935, 2860, 1689, 1605, 1588, 1518, 1388, 1378, 1254, 1230, 1003, 927 , 846. ${ }^{1} \mathrm{H}-\mathrm{NMR}\left(200 \mathrm{MHz}, \mathrm{CDCl}_{3}\right)$ : $1.31(d d d, J=4.2,6.8,8.2,1 \mathrm{H}) ; 1.63(d d d, J=4.4,5.2$, 9.4, $1 \mathrm{H}) ; 2.15(d d d, J=4.2,5.2,8.2,1 \mathrm{H}) ; 2.29(s, 3 \mathrm{H}) ; 2.49(d d d, J=4.0,6.6,9.0,1 \mathrm{H})$; $3.87(s, 3 \mathrm{H}) ; 5.12(s, 2 \mathrm{H}) ; 6.52-6.66(m, 2 \mathrm{H}) ; 6.78(d, J=8.2,1 \mathrm{H}) ; 7.25-7.44(m, 5 \mathrm{H})$. ${ }^{13} \mathrm{C}-\mathrm{NMR}\left(50 \mathrm{MHz}, \mathrm{CDCl}_{3}\right): 18.9 ; 28.8 ; 30.7 ; 32.7 ; 56 ; 71.2 ; 110.6 ; 114.4 ; 117.8 ; 127.2 ;$ 127.7; 128.4; 133.5; 137.2; 146.9; 149.7; 206.7 (CO). ESI-MS (40 eV): $297\left(7,[M+1]^{+}\right), 253$ (20.5), 219 (23.5), 205 (44.5), 189 (37), 158 (16.5), 91 (53). 
1-\{2-[3-Methoxy-4-(prop-2-en-1-yloxy)phenyl]cyclopropyl\}ethanone (3i). Yield: 69\%. Oil. IR (KBr): 2923, 1695, 1590, 1517, 1396, 1257, 1230, 1141, 1023, 929. ${ }^{1} \mathrm{H}-\mathrm{NMR}(200 \mathrm{MHz}$, $\left.\mathrm{CDCl}_{3}\right): 1.34(d d d, J=4.2,6.6,8.2,1 \mathrm{H}) ; 1.64(d d d, J=4.2,5.2,9.2,1 \mathrm{H}) ; 2.16(d d d, J=4.0$, $5.2,8.2,1 \mathrm{H}) ; 2.30(s, 3 \mathrm{H}) ; 2.48(d d d, J=4.0,6.6,9.2,1 \mathrm{H}) ; 3.86(s, 3 \mathrm{H}) ; 4.56-4.60(m, 2$ $\mathrm{H}) ; 5.24-5.44(m, 2 \mathrm{H}) ; 5.97-6.14(m, 1 \mathrm{H}) ; 6.58-6.65(m, 2 \mathrm{H}) ; 6.79(d, J=8.2,1 \mathrm{H})$.

${ }^{13} \mathrm{C}-\mathrm{NMR}\left(50 \mathrm{MHz}, \mathrm{CDCl}_{3}\right): 18.8 ; 28.7 ; 30.7 ; 32.7 ; 55.9 ; 70 ; 110.4 ; 113.7 ; 117.8 ; 133.2$; 133.3; 146.8; 149.5; 206.7 (CO). ESI-MS (10 eV): $247\left(100,[M+1]^{+}\right)$.

\section{1-(2-\{3-Methoxy-4-[(2-methylprop-2-en-1-yl)oxy]-phenyl\}cyclopropyl)ethanone}

Yield: 72\%. Oil. IR (KBr): 2922, 1694, 1591, 1516, 1393, 1264, 1229, 1139, 1035, 903. ${ }^{1} \mathrm{H}-\mathrm{NMR}\left(200 \mathrm{MHz}, \mathrm{CDCl}_{3}\right): 1.34(d d d, J=4.2,6.8,8.2,1 \mathrm{H}) ; 1.62(d d d, J=4.2,5.0,9.6,1$ $\mathrm{H}) ; 1.81(s, 3 \mathrm{H}) ; 2.16(d d d, J=4.0,5.2,8.2,1 \mathrm{H}) ; 2.30(s, 3 \mathrm{H}) ; 2.48(d d d, J=4.0,6.6,9.2,1$ $\mathrm{H}) ; 3.86(s, 3 \mathrm{H}) ; 4.48(s, 2 \mathrm{H}) ; 5.02(d, J=20.0,2 \mathrm{H}) ; 6.56-6.65(m, 2 \mathrm{H}) ; 6.79(d, J=8.2$, $1 \mathrm{H}) .{ }^{13} \mathrm{C}-\mathrm{NMR}\left(50 \mathrm{MHz}, \mathrm{CDCl}_{3}\right): 18.7 ; 19.2 ; 28.7 ; 30.6 ; 32.6 ; 55.9 ; 72.8 ; 110.5 ; 112.5$; 113.9; 117.8; 133.1; 140.8; 147; 149.5; $206.6(\mathrm{CO})$. ESI-MS (10 eV): $261\left(100,[M+1]^{+}\right)$.

\section{X-Ray Diffraction Experiment}

The diffraction data for molecule 3c were collected at r.t. on Agilent Gemini $S$ diffractometer equipped with $\mathrm{Cu} K_{\alpha}$ radiation $(\lambda=1.54184 \AA)$. Data reduction and empirical absorption correction were performed with CrysAlisPro. ${ }^{49}$ The crystal structure was solved by direct methods, using Sir2002 $2^{[50]}$ and refined using SHELXL ${ }^{[51]}$ by full-matrix least-squares on $F^{2}$. 
All H-atoms were placed geometrically $[\mathrm{C}-\mathrm{H}=0.93-0.98 \AA]$ and refined using the riding model with isotropic displacement parameters set to 1.2 or 1.5 times the $U_{\text {eq }}$ values of the parent C-atoms. Crystallographic data and refinement parameters are listed in Table S1. The software used for the preparation of the materials for publication: WinGX, ${ }^{52}$ Mercury, ${ }^{53}$ PLATON, ${ }^{54}$ PARST. $^{55}$

\section{Antimicrobial Activity}

Antimicrobial activities of tested compounds were evaluated against ten microorganisms, including five strains of bacteria: Staphylococcus aureus (ATCC 25923), B. subtilis (ATCC 6633), B. cereus (ATCC 10987), E. coli (ATCC 25922), and Proteus mirabilis (ATCC 29906) and five species of fungi: Aspergillus flavus (ATCC 9170), Aspergillus fumigatus (ATCC 1022), C. albicans (ATCC 10259), Penicillium purpurescens (ATCC 48987), and Penicillium verucosum (ATCC 48959) obtained from the American Type Culture Collection (ATCC).

The bacteria isolates were picked from overnight cultures in Mueller-Hinton agar and the suspensions were prepared in sterile distilled water by adjusting the turbidity to match 0.5 McFarland standards to approximately $10^{8} \mathrm{CFU} / \mathrm{ml}$. The fungal suspensions were prepared from 3- to 7-day-old cultures that grown on a potato dextrose agar except for C. albicans that was maintained on Sabourad dextrose (SD) agar. The spores were rinsed with sterile distilled 
water, used to determine turbidity spectrophotometrically at $530 \mathrm{~nm}$, and then further diluted to approximately $10^{6} \mathrm{CFU} / \mathrm{ml}$, following the procedure recommended by NCCLS. $\underline{56}$

The 96-well microtiter assay using resazurin as the indicator of cell growth, 57 was employed for the determination of the $M I C$ of the active components. The starting solutions of tested compounds were obtained by dissolving it in 5\% DMSO. The twofold serial dilutions of tested compounds were made in a concentration range from 20 to $0.004 \mathrm{mg} / \mathrm{ml}$ in sterile 96-well plates containing Mueller Hinton broth for bacterial cultures and a SD broth for fungal cultures. Then, fungal or bacterial suspensions were added to each well and finally, resazurin solution was added as an indicator to each well. The plates were prepared in triplicate, and placed in an incubator set at $37^{\circ} \mathrm{C}$ for $18-24 \mathrm{~h}$. The $M I C$ was determined visually and defined as the lowest concentration of tested compounds preventing resazurin color change from blue to pink. Streptomycin and ketoconazole were used as positive controls while 5\% DMSO was used as a negative control.

\section{Cytotoxicity}

Cells and Cell Culture. Human epithelial carcinoma HeLa cells, human lung carcinoma A549 cells and human colon carcinoma LS174 cells and non-malignant human lung fibroblast cell line (MRC5) were obtained from American Type Culture Collection (Manassas, VA, USA). All cancer cell lines were cultured as a monolayer in the RPMI 1640 nutrient medium, with 
$10 \%$ (inactivated at $56^{\circ} \mathrm{C}$ ) $\mathrm{FBS}, 3 \mathrm{mM}$ of 1 -glutamine, and antibiotics, at $37^{\circ} \mathrm{C}$ in humidified air atmosphere with $5 \% \mathrm{CO}_{2}$.

In vitro Cytotoxic Assay. In vitro assay for cytotoxic activity of tested compounds was performed when the cells reached $70-80 \%$ of confluence. Stock solution $(50 \mathrm{mg} / \mathrm{ml})$ of compounds was dissolved in corresponding medium to the required working concentrations. Neoplastic HeLa cells (5000 cells per well), A549 cells (5000 cells per well), and LS174 cells (5000 cells per well) as well as non-cancerous MRC5 (5000 cells per well) were seeded into 96-well microtiter plates, and $24 \mathrm{~h}$ later, after cell adherence, five different, double diluted concentrations of investigated compounds were added to the wells. Final compounds concentrations were $200,100,50,25$, and $12.5 \mu \mathrm{g} / \mathrm{ml}$ except for the control wells, where only nutrient medium was added. The cultures were incubated for the next $72 \mathrm{~h}$. The effect on cancer cell survival was determined $72 \mathrm{~h}$ after the addition of tested compounds, by the MTT test. 58 Briefly, $20 \mu \mathrm{l}$ of MTT solution ( $5 \mathrm{mg} / \mathrm{ml} \mathrm{PBS}$ ) was added to each well and incubated for a further $4 \mathrm{~h}$ at $37{ }^{\circ} \mathrm{C}$ in $5 \% \mathrm{CO}_{2}$ and humidified air. Subsequently, $100 \mu \mathrm{l}$ of $10 \% \mathrm{SDS}$ was added to solubilize the formazan crystals formed from MTT after the conversion by mitochondrial dehydrogenases of viable cells. Absorbencies proportional to the number of viable cells were measured using a microplate reader (Multiskan EX, Thermo Scientific, Finland) at $570 \mathrm{~nm}$. Each experiment was performed in triplicate and independently repeated at least four times. 


\section{Supplementary Material}

Supporting information for this article is available on the WWW under https://doi.org/10.1002/cbdv.201700077

\section{Acknowledgements}

The authors are grateful to the Ministry of Education, Science and Technological Development of the Republic of Serbia (Grant 172034) for financial support.

\section{Author Contribution Statement}

A. Z. Burmudžija, J. M. Muškinja, and Z. R. Ratković conceived and designed the chemistry experiments and wrote the article. M. M. Kosanić and B. R. Ranković performed the microbiological experiments. S. B. Novaković performed the X-ray experiments and discussion. S. B. Đorđević performed the structural measurement experiments. T. P. Stanojković and D. D. Baskić performed the cytotoxic experiments and discussion. 


\section{References:}

1. Varga, L., Nagy, T., Kövesdi, I., Benet-Bucholz, J., Dormán, G., Ürge, L., Darvas, F., ‘ Solution-Phase Parallel Synthesis of 4,6-Diaryl-pyrimidine-2-ylamines and 2-Amino-5,5-disubstituted-3,5-dihydro-imidazol-4-ones via a Rearrangement', Tetrahedron $2003,59,655-662$.

2. Kalirajan, R., Sivakumar, S. U., Jubie, S., Gowramma, B., Suresh, B., ' Synthesis and Biological Evaluation of Some Heterocyclic Derivatives of Chalcones', Int. J. ChemTech Res. $2009,1,27-34$.

3. Lin, S., Wei, Y., Liang, F., ' Cyanation of $\alpha, \beta$-Unsaturated Enones by Malononitrile in Open Air Under Metal-Catalyst-Free Conditions', Chem. Commun. 2012, 48, 9879 - 9881.

4. Samshuddin, S., Narayana, B., Sarojini, B. K., Yathirajan, H. S., Raghavendra, R., ' Synthesis, Characterization and Biological Evaluation of Functionalized Derivatives of Versatile Synthon 4,4'-Difluoro Chalcone', Der Pharma Chemica 2012, 4, 1445 - 1457.

5. Corey, E. J., Chaykovsky, M., ' Dimethyloxosulfonium methylide $\left(\left(\mathrm{CH}_{3}\right)_{2} \mathrm{SOCH}_{2}\right)$ and Dimethylsulfonium methylide $\left(\left(\mathrm{CH}_{3}\right)_{2} \mathrm{SCH}_{2}\right)$. Formation and Application to Organic Synthesis', J. Am. Chem. Soc. 1965, 87, $1353-1364$.

6. Motohashi, N., Yamagami, C., Tokuda, H., Konoshima, T., Okuda, Y., Okuda, M., Mukainaka, T., Nishino, H., Saito, Y., ' Inhibitory Effects of Dehydrozingerone and Related Compounds on 12-O-tetradecanoylphorbol-13-acetate Induced Epstein-Barr Virus Early Antigen Activation', Cancer Lett. 1998, 134, 37 - 42. 
7. Adams, B. K., Ferstl, E. M., Davis, M. C., Herold, M., Kurtkaya, S., Camalier, R. F., Hollingshead, M. G., Kaur, G., Sausville, E. A., Rickles, F. R., Snyder, J. P., Liotta, D. C., Shoji, M., ' Synthesis and Biological Evaluation of Novel Curcumin Analogs as Anticancer and Anti-Angiogenesis Agents', Bioorg. Med. Chem. 2004, 12, 3871 - 3883.

8. Ishida, J., Ohtsu, H., Tachibana, Y., Nakanishi, Y., Bastow, K. F., Nagi, M., Wang, H.-K., Itokawa, H., Lee, K.-H., ‘ Antitumor Agents. Part 214: Synthesis and Evaluation of Curcumin Analogues as Cytotoxic Agents', Bioorg. Med. Chem. 2002, 10, 3481 - 3487.

9. Levai, A., ' Synthesis of 2-Pyrazolines by the Reactions of $\alpha, \beta$-Unsaturated Aldehydes, Ketones, and Esters with Diazoalkanes, Nitrile, Imines, and Hydrazines', J. Heterocycl. Chem. $2002,39,1-13$.

10. Kissane, M., Maguire, A. R., 'Asymmetric 1,3-Dipolar Cycloadditions of Acrylamides', Chem. Soc. Rev. 2010, 39, $845-883$.

11. Ali, M. A., Shaharyar, M., "Discovery of Novel Phenoxyacetic Acid Derivatives as Antimycobacterial Agents', Bioorg. Med. Chem. 2007, 15, 1896 - 1902.

12. Ratković, Z., Muškinja, J., Burmudžija, A., Ranković, B., Kosanić, M., Bogdanović, G. A., Simović-Marković, B., Nikolić, A., Arsenijević, N., Đorđević, S., Vukićević, R. D., ‘ Dehydrozingerone Based 1-Acetyl-5-aryl-4,5-dihydro-1 $H$-pyrazoles: Synthesis, Characterization and Anticancer Activity', J. Mol. Struct. 2016, 1109, 82 - 88.

13. Wessjohann, L. A., Brandt, W., Thiemann, T., ' Biosynthesis and Metabolism of Cyclopropane Rings in Natural Compounds', Chem. Rev. 2003, 103, 1625 - 1648. 
14. Witvrouw, M., Pannecouque, C., De Clercq, E., Fernández-Alvarez, E., Marco, J. L., ‘ Inhibition of Human Immunodeficiency Virus Type (HIV-1) Replication by some Diversely Functionalized Spirocyclopropyl Derivatives', Arch. Pharm. 1999, 332, 163 - 166.

15. Pinto, I. L., West, A., Debouck, C. M., DiLella, A. G., Gorniak, J. G., O'Donnell, K. C., O'Shannessy, D. J., Patel, A., Jarvest, R. L., ‘ Novel, Selective Mechanism-Based Inhibitors of the Herpes Proteases', Bioorg. Med. Chem. Lett. 1996, 6, 2467 - 2472.

16. Faust, R., ' Fascinating Natural and Artificial Cyclopropane Architectures', Angew. Chem., Int. Ed. 2001, 40, $2251-2253$.

17. Boger, D. L., Hughes, T. V., Hedrick, M. P., ' Synthesis, Chemical Properties, and Biological Evaluation of CC-1065 and Duocarmycin Analogues Incorporating the 5-Methoxycarbonyl-1,2,9,9a-tetrahydrocyclopropa[c]benz[e]indol-4-one Alkylation Subunit', J. Org. Chem. 2001, 66, $2207-2216$.

18. Yoshida, S., Rosen, T. C., Meyer, O. G. J., Sloan, M. J., Ye, S., Haufe, G., Kirk, K. L., ‘ Fluorinated Phenylcyclopropylamines. Part 3: Inhibition of Monoamine Oxidases A and B', Bioorg. Med. Chem. 2004, 12, $2645-2652$.

19. Patrick, T. B., Neal, B. E., ' Synthesis of a Cyclopropane Ring Fluorinated Pyrethroid Derivativive', Synlett 1996, 12, 1227 - 1228.

20. Elliott, M., Janes, N. F., Spanner, J. A., ' The Pyrethrins and Related Compounds. XVII. Preparation of the Insecticide Bioresmethrin (5-Benzyl-3-furylmethyl (+)-trans-chrysanthemate) and Related Compounds Labelled with Deuterium or Tritium on the Furan Ring', Pestic. Sci. 1973, 4, $677-681$. 
21. Noolvi, M. N., Patel, H. M., Singh, N., Gadad, A. K., Cameotra, S. S., Badiger, A., ' Synthesis and Anticancer Evaluation of Novel 2-Cyclopropylimidazo [2,1-b][1,3,4]-thiadiazole Derivatives', Eur. J. Med. Chem. 2011, 46, $4411-4418$.

22. Nie, Z., Perretta, C., Erickson, P., Margosiak, S., Lu, J., Averill, A., Almassy, R., Chu, S., ‘ Structure-Based Design and Synthesis of Novel Macrocyclic Pyrazolo[1,5- $a][1,3,5]$ triazine Compounds as Potent Inhibitors of Protein Kinase CK2 and Their Anticancer Activities', Bioorg. Med. Chem. Lett. 2008, 18, $619-623$.

23. Tung, N. H., Minh, C. V., Ha, T. T., Kiem, P. V., Huong, H. T., Dat, N. T., Nhiem, N. X., Tai, B. H., Hyun, J.-H., Kang, H.-K., Kim, Y. H., ' $\mathrm{C}_{29}$ Sterols with a Cyclopropane Ring at C-25 and 26 from the Vietnamese Marine Sponge Ianthella sp. and Their Anticancer Properties', Bioorg. Med. Chem. Lett. 2009, 19, $4584-4588$.

24. Jonnalagadda, S. S., Haar, E., Hamel, E., Lin, C. M., Magarian, R. A., Day, B. W., ' Synthesis and Biological Evaluation of 1,1-Dichloro-2,3-diarylcyclopropanes as Antitubulin and Anti-Breast Cancer Agents', Bioorg. Med. Chem. 1997, 5, 715 - 722.

25. Overacre, L. B., Magarian, R. A., ' Synthesis and Biological Evaluation of Cyclopropyl Analogs of the Antiestrogen MER 25', Bioorg. Chem. 1998, 26, 15 - 31.

26. Ty, N., Kaffy, J., Arrault, A., Thoret, S., Pontikis, R., Dubois, J., Morin-Allory, L., Florent, J.-C.,' Synthesis and Biological Evaluation of cis-Locked Vinylogous Combretastatin-A4 Analogues: Derivatives with a Cyclopropyl-Vinyl or a Cyclopropyl-Amide Bridge', Bioorg. Med. Chem. Lett. 2009, 19, 1318 - 1322. 
27. Fürst, R., Zupkó, I., Berényi, Á., Ecker, G. F., Rinner, U., ‘ Synthesis and Antitumor-Evaluation of Cyclopropyl-Containing Combretastatin Analogs', Bioorg. Med. Chem. Lett. 2009, 19, $6948-6951$.

28. Moody, C. J., Norton, C. L., Slawin, A. M. Z., Taylor, S., ' Cyclopropyl Indolequinones: Mechanistic Probes for Bioreductive Anticancer Drug Action', Anti-cancer Drug Design $1998,13,611-634$.

29. De, P., Baltas, M., Lamoral-Theys, D., Bruyère, C., Kiss, R., Bedos-Belval, F., Saffon, N., ‘ Synthesis and Anticancer Activity Evaluation of 2(4-Alkoxyphenyl)cyclopropyl hydrazides and Triazolo Phthalazines', Bioorg. Med. Chem. 2010, 18, 2537 - 2548.

30. Baker, J. T., Borris, R. P., Carté, B., Cordell, G. A., Soejarto, D. D., Cragg, G. M., Gupta, M. P., Iwu, M. M., Madulid, D. R., Tyler, V. E., ' Natural Product Drug Discovery and Development: New Perspectives on International Collaboration', J. Nat. Prod. 1995, 58, 1325 $-1357$.

31. Joksović, M., Ratković, Z., Vukićević, M., Vukićević, R. D., ‘ Synthesis of 1H-3-Ferrocenyl-1-phenyl-4-substituted Pyrazoles', Synlett 2006, 2581 - 2584.

32. Damljanović, I., Vukićević, M., Radulović, N., Palić, R., Ellmerer, E., Ratković, Z., Joksović, M., Vukićević, R. D., ' Synthesis and Antimicrobial Activity of Some New Pyrazole Derivatives Containing a Ferrocene Unit', Bioorg. Med. Chem. Lett. 2009, 19, 1093 - 1096.

33. Damljanović, I., Čolović, M., Vukićević, M., Manojlović, D., Radulović, N., Wurst, K., Laus, G., Ratković, Z., Joksović, M., Vukićević, R. D., ‘ Synthesis, Spectral Characterization and Electrochemical Properties of $1 \mathrm{H}-3-\left(o^{-}, \quad, \quad m-\quad\right.$ and 
p-ferrocenylphenyl)-1-phenylpyrazole-4-carboxaldehydes', J. Organomet. Chem. 2009, 694, $1575-1580$.

34. Ratković, Z., Juranić, Z. D., Stanojković, T., Manojlović, D., Vukićević, R. D., Radulović, N., Joksović, M. D., ' Synthesis, Characterization, Electrochemical Studies and Antitumor Activity of Some New Chalcone Analogues Containing Ferrocenyl Pyrazole Moiety', Bioorg. Chem. 2010, 38, $26-32$.

35. Smith, L. R., " Rheosmin ("Raspberry Ketone") and Zingerone, and Their Preparation by Crossed Aldol-Catalytic Hydrogenation Sequences', Chem. Educator 1996, 1(3), 1 - 18.

36. Gerkin, R. E., ' Hydrogen Bonding and Ring Asymmetry in a Substituted Cyclopropane: (+)-trans-(1S,2S)-2-Phenylcyclopropanecarboxylic Ccid at 208K', Acta Crystallogr., Sect. C $1997,53,1280-1282$.

37. Gerkin, R.E., 'Hydrogen Bonding and Ring Asymmetry in (土)-cis-2-Phenylcyclopropanecarboxylic Acid', Acta Crystallogr., Sect. C 1997, 53, 1989 1991.

38. Zhang, M.-X., Eaton, P. E., ' $\mathrm{BuMgNiPr}_{2}$ : A New Base for Stoichiometric, Position-Selective Deprotonation of Cyclopropane Carboxamides and Other Weak CH Acids', Angew. Chem., Int. Ed. 2002, 41, $2169-2171$.

39. Boer, J. S. A. M., Loopstra, B. O., Stam, C. H., ' The Influence of Substituents on the Geometry of the Cyclopropane Ring: III. The Molecular and Crystal Structure of Phenylcyclopropane', Rec. Trav. Chim. Pays-Bas 1987, 106, 537 - 538.

40. Muškinja, J., Burmudžija, A., Ratković, Z., Ranković, B., Kosanić, M., Bogdanović, G. A., Novaković, S. B., ' Ferrocenyl Chalcones with $O$-Alkylated Vanillins: Synthesis, Spectral 
Characterization, Microbiological Evaluation, and Single-Crystal X-ray Analysis', Med. Chem. Res. 2016, 25, $1744-1753$.

41. Ristić, S., Ranković, B., Kosanić, M., Stanojković, T., Stamenković, S., Vasiljević, P., Manojlović, I., Manojlović, N., ' Phytochemical Study and Antioxidant, Antimicrobial and Anticancer Activities of Melanelia subaurifera and Melanelia fuliginosa Lichens', J. Food Sci. Technol. 2016, 53, $2804-2816$.

42. Aleksić, V., Knežević, P., 'Antimicrobial and Antioxidative Activity of Extracts and Essential Oils of Myrtus communis L.', Microbiol. Res. 2014, 169, 240 - 254.

43. Kosanić, M., Ranković, B., Dašić, M., ' Mushrooms as Possible Antioxidant and Antimicrobial Agents', Iran. J. Pharm. Res. 2012, 11, 1095 - 1102.

44. Loev, B., Dawson, C. R., ' Alkenylphenols Related to the Poison Ivy Principle. An Improved Method of Synthesis Involving the Na-Butanol Cleavage of Benzyl Ethers', J. Am. Chem. Soc. $1956,78,6095-6098$.

45. Tatsuzaki, J., Bastow, K. F., Nakagawa-Goto, K., Nakamura, S., Itokawa, H., Lee, K.-H., ‘ Dehydrozingerone, Chalcone, and Isoeugenol Analogues as in Vitro Anticancer Agents', J. Nat. Prod. 2006, 69, $1445-1449$.

46. Katritzky, A. R., Long, Q., He, H.-Y., Qiua, G., Wilcox, A. L., ' Preparation of 2-Alkoxy-5-methoxybenzaldehydes and 2-Ethoxy-5-alkoxybenzaldehydes', Arkivoc 2000, (vi), $868-875$.

47. Tatsuzaki, J., Nakagawa-Goto, K., Tokuda, H., Lee, K., ' Cancer Preventive Agents 10. Prenylated Dehydrozingerone Analogs as Potent Chemopreventive Agents', J. Asian Nat. Prod. Res. 2010, 12, $227-232$. 
48. Nongkhlaw, R. L., Nongrum, R., Nongkynrih, I., Vattakunnel, F. M., Myrboh, B., ' Novel Synthesis of Substituted Cyclopropane Acetic Acid Ethyl Esters from Cyclopropyl Alkyl Ketones', Ind. J. Chem., Sect. B, 2005, 44, $1054-1057$.

49. Agilent, CrysAlis PRO, Agilent Technologies, Yarnton, Oxfordshire, England, 2013.

50. Burla, C. M., Camalli, M., Carrozzini, B., Cascarano, G. L., Giacovazzo, C., Polidori, G., Spagna, R., ‘ SIR2002: The Program', J. Appl. Crystallogr. 2003, 36, 1103.

51. Sheldrick, G. M., ‘ A Short History of SHELX’, Acta Crystallogr., Sect. A 2008, 645, 112 122.

52. 52 Farrugia, L. J., ' WinGX Suite for Small-Molecule Single-Crystal Crystallography', J. Appl. Crystallogr. 1999, 32, $837-838$.

53. Macrae, C. F., Edgington, P. R., McCabe, P., Pidcock, E., Shields, G. P., Taylor, R., Towler, M., Streek, J., J. Appl. Crystallogr. 2006, 39, $453-457$.

54. Spek, A. L., ' Single-Crystal Structure Validation with the Program PLATON', J. Appl. Crystallogr. 2003, 36, $7-13$.

55. Nardelli, M., 'PARST95-An Update to PARST: A System of Fortran Routines for Calculating Molecular Structure Parameters from the Results of Crystal Structure Analyses', J. Appl. Crystallogr. 1995, 28, 659.

56. NCCLS (National Commitee for Clinical Laboratory Standards). Reference method for broth dilution antifungal susceptibility testing of conidium-forming filamentous fungi: Proposed Standard M38-P. NCCLS, Wayne, PA, USA, 1998. 
57. Sarker, S. D., Nahar, L., Kumarasamy, Y., ' Microtitre Plate-Based Antibacterial Assay Incorporating Resazurin as an Indicator of Cell Growth, and Its Application in the In Vitro Antibacterial Screening of Phytochemicals', Methods 2007, 42, 321 - 324.

58. Mosmann, T., ' Rapid Colorimetric Assay for Cellular Growth and Survival: Application to Proliferation and Cytotoxicity Assays', J. Immunol. Methods 1983, 65, 55 - 63. 